\title{
Demokratie stärken, Engagement fördern
}

\section{Die Bundesprogramme gegen Rechtsextremismus}

\author{
Hermann Kues
}

\section{Dr. Hermann Kues ist}

Parlamentarischer Staatssekretär im

Bundesministerium für Familie,

Senioren, Frauen und Jugend.

Internet http://www.bmfsfj.de
Das Bundesministerium für Familie, Senioren, Frauen und Jugend hat einige Programme aufgelegt, die dem Rechtsextremismus in Schule, Verein und der Jugendhilfe wehren sollen. Dazu gehört auch ein Beratungsnetzwerk, das Diensten und Einrichtungen bei Konflikten zur Seite steht.

Fremdenfeindliche Schläger, die Menschen durch die Straßen jagen. Hakenkreuzgeschmiere auf jüdischen Friedhöfen. Rassistische Witze in der Schulpause. Und Zulauf für rechtsextreme Parteien, die bei demokratischen Wahlen auf Stimmenfang gehen, mit dem Ziel, die Demokratie abzuschaffen. Das alles zeigt: Unsere Gesellschaft muss ihre Grundwerte auch heute, 60 Jahre nach der Einführung des Grundgesetzes, couragiert verteidigen. Unsere Kinder, die Gesellschaft und Staat in Zukunft bestimmen und gestalten, sollen diese Werte frühzeitig nicht nur kennen sondern auch schätzen lernen. Das Bundesjugendministerium hat sich daher zum Ziel gesetzt, gerade bei jungen Menschen den Sinn für Demokratie, Toleranz und Weltoffenheit zu fördern.

»VIELFALT TUT GUT. Jugend für Vielfalt, Toleranz und Demokratie « heißt das präventiv-pädagogisch Bundesprogramm, das das Ministerium am 1. Januar 2007 gestartet hat. Ziel ist es, Kinder und Jugendliche gegen den Einfluss der rechtsextremen Szene zu stärken. Auch die örtlichen Meinungsträgerinnen und Meinungsträger wie der Vereinsvorsitzende, die Jugendgruppenleiterin oder der Sporttrainer werden angesprochen, sich verstärkt für kulturelle Vielfalt und Toleranz einzusetzen. Das Programm richtet sich vor allem an junge Menschen, rechtsextremistisch gefährdete Jugendliche, Migrantinnen und Migranten sowie Eltern, Erzieherinnen und Erzieher sowie Lehrerinnen und Lehrer. VIELFALT TUT GUT, für das der Bund bis 2010 jährlich 19 Millionen Euro bereit stellt, setzt dabei auf zwei Schwerpunkte: Lokale Aktionspläne und Modellprojekte.

\section{Lokale Aktionspläne}

Durch Lokale Aktionspläne werden Städte und Landkreise im Kampf gegen rechtsextreme und fremdenfeindliche Aktivitäten unterstützt. Im Aktionsplan arbeiten dabei Verwaltung und Vereine, Kirchen und Initiativen, Verbände und Beratungsstellen, engagierte Bürgerinnen und Bürger sowie Jugendliche zusammen. Gemeinsam entwickeln sie Strategien, wie sie dem Einfluss und den Aktivitäten rechtsextremer Gruppen und Parteien in ihrer Region begegnen und Toleranz fördern können. So werden auch einzelne Aktionen an Schulen, von Vereinen oder Bürgerinitiativen gebündelt und vernetzt. Bundesweit fördert das Programm bis 2010 insgesamt 90 Lokale Aktionspläne. Bereits jetzt haben diese Aktionspläne weit mehr als 2.000 einzelne Projekte, Workshops und Veranstaltungen unterstützt. Auch die Träger der Freien Wohlfahrtspflege, der Sozialen Arbeit sowie der Jugendhilfe engagieren sich, wie das Beispiel der Stadt Braunschweig zeigt.

Ziel des Lokalen Aktionsplans in Braunschweig ist es, die dauerhafte Integration von Migrantinnen und Migranten zu fördern und so Fremdenfeindlichkeit abzubauen. Der Plan wurde von Migrantenselbstorganisationen, Trägern der freien Jugendhilfe, Verwaltung und Wirtschaft sowie Arbeiterwohlfahrt und Caritas entwickelt. Bisher wurden mehr als 30 Projekte gestartet, wie beispielsweise die Imagekampagne für junge Migrantinnen und Migranten der Caritas. Das Projekt »Orient schmückt Okzident « der Arbeiterwohlfahrt hat einen anderen Ansatz: Mütter mit Migrationshintergrund sollen stärker in ihr direktes soziales Umfeld eingebunden werden. Konkret heißt das: Deutsche und Mütter aus Migrantenfamilien erlernen gemeinsam und vonein- 
ander unterschiedliche kunsthandwerkliche Techniken. Die so entstandenen Werke stellen die Organisatoren in den Kindergärten aus und können von den Kindern dort genutzt werden.

Ein anderes Beispiel ist der Lokale Aktionsplan im Brandenburger Landkreis Ostprignitz-Ruppin. Der Aktionsplan basiert auf den Ergebnissen einer Regionalkonferenz, an der sich auch die Wohl-
Workshops lernen sie die eigenen und fremden Wertesysteme und kulturellen Identitäten kennen und hinterfragen sie. Was die mehr als 80 Kinder und Jugendlichen im Alter von 5 bis 25 Jahren in ihren Arbeitsgruppen zu Streetdance, Hiphop, Malen, Gedichte, Stockkampf oder Diabolo gelernt haben, zeigen sie anschließend in einer gemeinsamen Zirkusaufführung. Die Ergebnisse des Modell-

\section{»Berater bieten bei rechtsextremistischen Aktivitäten ihre Unterstützung an"}

fahrtsverbände beteiligten. Sie haben sich zu einem Trägerverbund zusammengeschlossen, der auch als Antragsteller auftritt. So war es beispielsweise möglich, mit einer Fortbildungsinitiative alle von den Verbänden getragenen Kindertagesstätten im Landkreis zu erreichen. Die Erzieherinnen und Erzieher dort werden jetzt geschult, wie sie den Kindern Verständnis für Fremde und Toleranzbewusstsein vermitteln können. Der Erfolg der Initiative hat die Organisatoren dazu bewogen, eine ähnliche »konstatierte Aktion« für Eltern anzubieten.

\section{Modellprojekte}

Den zweiten Schwerpunkt von VIELFALT TUT GUT bilden die Modellprojekte. Die Projekte entwickeln und erproben neue Ansätze und Methoden in der pädagogischen Bildungsarbeit mit Kindern und Jugendlichen. Der Bund fördert sie für eine begrenzte Zeit und stößt damit neue Entwicklungen an. Ziel ist die Vermittlung demokratischer Werte, um Jugendliche gegen die Anziehungskraft der rechtsextremen Szene zu stärken. Derzeit laufen in ganz Deutschland mehr als 90 Projekte, wie das dreijährige Modellprojekt »Open space - Open mind, multiethnische Minderheiten im Mikrokosmos " des Kinder- und Jugendzirkus' Cabuwazi e. V. in Berlin. »Open space Open mind « zielt auf interkulturelle Verständigung der Kinder und Jugendliche mit und ohne Migrationshintergrund, die in der Nachbarschaft von Cabuwazi leben. In akrobatischen und künstlerischen projekts sollen in die Jugendhilfeplanung des Bezirks einfließen und nach dem Ende des Projekts als Arbeitshilfe veröffentlicht werden.

VIELFALT TUT GUT ist vor allem präventiv angelegt. In Deutschland werden jedoch fast täglich Menschen Opfer rechtsextremer Bedrohung oder Gewalt. Um in solchen Situationen schnell und direkt helfen zu können, hat das Bundesjugendministerium im Juli 2007 das Programm »kompetent. für Demokratie Beratungsnetzwerke gegen Rechtsextremismus « gestartet. Mit seiner Hilfe wurden seither gemeinsam mit den Bundesländern Beratungsnetzwerke aufgebaut, in denen Verwaltung, Jugend- und Sozialarbeit, Schule, Polizei und Verfassungsschutz, aber auch Kirchen, Wohlfahrtsverbände, Projekte, Initiativen und Netzwerke zusammenarbeiten. Bis 2010 fördert das Bundesjugendministerium die 16 landesweiten Netzwerke und Beratungsteams mit jährlich fünf Millionen Euro.

Die Berater bieten bei rechtsextremistischen, fremdenfeindlichen oder antisemitischen Aktivitäten oder Übergriffen den Betroffenen und den zuständigen Behörden ihre Unterstützung an. Gemeinsam mit den Menschen vor Ort entwickeln sie dabei Ideen, wie Konflikte in Zukunft selbstständig gelöst werden können. Wie sie dabei konkret vorgehen, zeigt ein Beispiel aus Bremen: Im Jugendfreizeithaus kapselt sich eine Clique mehr und mehr von den anderen Besucherinnen und Besuchern ab. Als Reaktion auf einen Angriff jugendlicher Migranten auf Mädchen aus der Gruppe hören die Jungen und Mädchen zwischen 14 und 17 Jahren Rechtsrock und machen mit Parolen wie "Ausländer raus « auf sich aufmerksam. Für die Mitarbeiterinnen und Mitarbeiter des Jugendhauses eine schwierige Situation - umso mehr, da im Umfeld bereits rechtsextreme Gruppen aktiv sind. Sie bitten daher die Beratungsstelle "pro aktiv gegen rechts - Mobile Beratung in Bremen und Bremerhaven « um Hilfe. Für die Berater ist klar: Nicht einfach die Musik abdrehen, sondern das Grundproblem angehen, muss die Strategie sein. An erster Stelle steht daher das Gespräch mit den Jugendlichen. Sie sollen sich nach und nach mit ihrer Einstellung auseinandersetzen und erkennen, dass es andere Lösungswege als Gewalt und Parolen gibt. Auch die Mitarbeiterinnen und Mitarbeiter im Jugendhaus erhalten Unterstützung: Das Beraterteam zeigt ihnen, wie sie zukünftig mit Provokationen und Gewalt umgehen können.

\section{Alle sind gefordert}

Mit ihren Programmen setzt die Bundesregierung im Kampf gegen den Rechtsextremismus in Deutschland ein klares Zeichen. Der Schutz unserer Kinder erfordert jedoch mehr als Modellprojekte und staatliche Fördermittel. Jeder einzelne ist gefordert, gegen Intoleranz und Fremdenfeindlichkeit Stellung zu beziehen. In der Auseinandersetzung mit dem Rechtsextremismus sind deshalb alle gesellschaftlichen Kräfte gefordert, um für die Werte unserer Demokratie einzustehen. 